\title{
The Anisotropic Glassy Properties of Decagonal Quasicrystals
}

\author{
Dragoş-Victor Anghel $^{1}$ and Dmitry V. Churochkin ${ }^{2}$ \\ ${ }^{1}$ Horia Hulubei National Institute for Physics and Nuclear Engineering, 30 Reactorului Street, P.O. Box MG-6, \\ Măgurele RO-077125, Jud. Ilfov, Romania \\ ${ }^{2}$ Department of Physics, Faculty of Mathematical and Physical Sciences, University of Chile, Avenue Blanco Encalada 2008, \\ Santiago, Chile
}

Correspondence should be addressed to Dragoş-Victor Anghel; dragos@theory.nipne.ro

Received 3 May 2013; Accepted 26 September 2013

Academic Editor: Robert Leisure

Copyright (c) 2013 D.-V. Anghel and D. V. Churochkin. This is an open access article distributed under the Creative Commons Attribution License, which permits unrestricted use, distribution, and reproduction in any medium, provided the original work is properly cited.

\begin{abstract}
We use an extended version of the standard tunneling model to explain the anisotropic sound absorption in decagonal quasicrystals. The glassy properties are determined by an ensemble of two level systems (TLSs), arbitrarily oriented. The TLS is characterized by a $3 \times 3$ symmetric tensor, $[T]$, which couples to the strain field, $[S]$, through a $3 \times 3 \times 3 \times 3$ tensor of coupling constants, $[R]$. The structure of $[R]$ reflects the symmetry of the quasicrystal. We also analyze the probability distributions of the elements of $[T]$ in this particular model for a better understanding of the characteristics of "isotropic" and "anisotropic" distributions of the ensemble of TLSs. We observe that the distribution of the elements is neither simple nor intuitive and therefore it is difficult to guess it $a$ priory, using qualitative arguments based on the symmetry properties.
\end{abstract}

\section{Introduction}

Despite almost four decades of study of the glassy properties materials [1-4], the nature of the two-level systems (TLS), the ubiquitous hypothetical microscopic entities that are held responsible for these properties, is still not known. As a general picture, it is accepted that they represent dynamic defects, which are atoms or groups of atoms that tunnel from one minimum energy configuration to another. But these atoms are in general not identified, and even in the cases when they are identified (like in crystals with defects), the TLS spectrum cannot be obtained based on a microscopic model.

The study of solids with anisotropic glassy properties is especially interesting since this brings additional information about the TLSs and requires a critical perspective on the standard tunneling model (STM).

To identify the origin of the TLS, the thermal and acoustical properties of a number of crystalline systems with defects [5-13] and quasicrystals [14-18] at low temperatures have been investigated thoroughly. As expected, the glass like properties in both the disordered crystalline system and in the quasicrystals were revealed. However, a crucial difference in comparison with ordinary amorphous solids which possess TLS excitations was detected. Namely, pronounced anisotropy in internal friction was clearly marked. As a consequence of that, the baffling physical problem about the possible origin of the anisotropy appeared. Indeed, there are two competitive ways for the explanation. First, the effect is explained by an anisotropy in the distribution over the "orientations" of the TLSs in the ensemble. The interaction between the TLS and the elastic field is described by the Hamiltonian

$$
H_{I}=\frac{1}{2}\left(\begin{array}{cc}
\delta & 0 \\
0 & -\delta
\end{array}\right) \text {, }
$$

where $\delta \equiv 2 \gamma_{i j} S_{i j}$ and $[S]$ is the strain field of the phononwe assume everywhere summation over the repeated indices. The symmetric second rank tensor $[\gamma]$ characterizes the TLS and its "deformability" under elastic strain. The anisotropy of the physical properties is a reflection of the values taken by the elements of $[\gamma]$, which are determined by the lattice symmetries. Bert et al. made a conjecture regarding these values to recover the anisotropic sound attenuation rates that they observed in decagonal quasicrystals [18]. 
In the second approach [19-21]-the one that we will employ in this paper-the TLS is characterized by a $3 \times 3$ symmetric tensor $[T]$ and the coupling between $[T]$ and $[S]$ is made through a forth rank tensor of coupling constants denoted by $[R]$. Explicitly, $\gamma_{i j} \equiv R_{k l i j} T_{k l}$ and $\delta \equiv[T]^{t}:[R]$ : $[S]$. The elements of $[T]$ are determined by a unit vector $\widehat{\mathbf{t}}$, which is the direction of the TLS, whereas the structure of $[R]$ is determined by the symmetries of the host material. In this model, even if the TLSs are isotropically oriented, the anisotropy of the system is imposed by the properties of the tensor of coupling constants.

We applied the second approach to the crystalline materials of different symmetries with embedded TLSs, assuming the isotropy of the orientations of the TLSs [22-25] and we calculated the attenuation of ultrasound waves of different polarizations and propagating in different crystallographic directions. This model describes not only in a simple way the asymmetries of the glassy properties but also allows us to make predictions about the relative attenuation rates of sound propagating in different directions.

In this paper, we apply the model of [19] to the attenuation of ultrasound waves in quasicrystals and we obtain the attenuation rates along different crystallographic directions expressed in terms of the components of the tensor of coupling constants. We apply our calculations to the experimental results of [18], which enable us to calculate the relations between some of the components of the coupling constants tensor.

In order to better understand the distribution of the TLSs in isotropic and anisotropic materials, we calculate the distribution of the elements of [T] for an isotropic distribution of unit vectors $\widehat{\mathbf{t}}$.

\section{The Anisotropy of the Glassy Properties}

Let us introduce the notations by presenting briefly the model. The Hamiltonian of the free TLS is

$$
H_{\mathrm{TLS}}=\frac{\Delta}{2} \sigma_{z}-\frac{\Lambda}{2} \sigma_{x} \equiv \frac{1}{2}\left(\begin{array}{cc}
\Delta & -\Lambda \\
-\Lambda & -\Delta
\end{array}\right),
$$

where $\Delta$ is called the asymmetry of the potential and $\Lambda$ the tunnel splitting. The eigenvalues of $H_{\mathrm{TLS}}$ are $\pm \epsilon / 2$, where $\epsilon \equiv$ $\sqrt{\Delta^{2}+\Lambda^{2}}$ is the excitation energy of this TLS. The ground state will be denoted by $|\downarrow\rangle$ and the excited state by $|\uparrow\rangle$. The interaction Hamiltonian of the TLS with the strain field is given by (1).

The parameters $\Delta$ and $\Lambda$ are distributed with the probability $P(\Delta, \Lambda)=P_{0} / \Lambda$, where $P_{0}$ is a constant. If expressed in terms of $\epsilon$ and $u \equiv \Lambda / \epsilon$, the probability distribution becomes $P(\epsilon, u)=P_{0} /\left(u \sqrt{1-u^{2}}\right)$.

As usual, we work in the abbreviated subscript notations and write $[S]$ and $[\gamma]$ as six-dimensional vectors: $\mathbf{S}=$ $\left(S_{11}, S_{22}, S_{33}, 2 S_{23}, 2 S_{13}, 2 S_{12}\right)^{t}$ and $\gamma=\left(\gamma_{11}, \gamma_{22}, \gamma_{33}, \gamma_{23}, \gamma_{13}\right.$, $\left.\gamma_{12}\right)^{t}$, where the superscript $t$ denotes the transpose of a matrix or a vector.

As stated before, $\gamma_{i j}=R_{k l i j} T_{k l}$. Assuming that the TLS is characterized by a direction in space, $\widehat{\mathbf{t}}$, the tensor $\mathbf{T}$ is formed of the components of $\widehat{\mathbf{t}}$ [19]. In abbreviated subscript notations $\mathbf{T} \equiv\left(t_{x}^{2}, t_{y}^{2}, t_{x}^{2}, 2 t_{y} t_{z}, 2 t_{z} t_{x}, 2 t_{x} t_{y}\right)^{T}, R_{i j k l}$ becomes $R_{I J}$, and $\gamma \equiv[R]^{t} \cdot \mathbf{T}[19]$. The structure of $[R]$ is determined by the symmetries of the lattice, since $\delta$ is a scalar and it should be invariant under coordinates transformations, whereas $[R]$ should be invariant under the symmetry transformations that leaves the lattice invariant [19].

The absorption rate of a phonon-with wavenumber $\mathbf{k}$ and polarization $\sigma$-by a TLS is $[19,21,22,24]$

$$
\Gamma_{\mathbf{k} \sigma}(\hat{\mathbf{t}})=\frac{2 \pi}{\hbar} \frac{\Lambda^{2} n_{\mathbf{k} \sigma}}{\epsilon^{2}}\left|\mathbf{T}^{t} \cdot[R] \cdot \mathbf{S}_{\mathbf{k} \sigma}\right|^{2} \delta(\epsilon-\hbar \omega)
$$

The main characteristic of the TLS-phonon interaction is contained in the quantity $M_{\mathbf{k}, \sigma}(\mathbf{t}) \equiv \mathbf{T}^{t} \cdot[R] \cdot \mathbf{S}_{\mathbf{k} \sigma}$, which bears an intrinsic anisotropy through the matrix $[R]$, on which the symmetries of the lattice are imposed.

The average scattering rate of a phonon by the ensemble of TLSs is obtained by averaging $\Gamma_{\mathbf{k} \sigma}(\widehat{t})$ over $\epsilon, \Lambda$, and $\widehat{\mathbf{t}}$. In this way we get the total phonon absorption rate:

$$
\tau_{\mathbf{k} \sigma}^{-1}=\frac{2 \pi}{\hbar} P_{0}\left\langle\left|M_{\mathbf{k} \sigma}(\widehat{\mathbf{t}})\right|^{2}\right\rangle n_{\mathbf{k} \sigma} \tanh \left(\frac{\epsilon}{2 k_{\mathrm{B}} T}\right)
$$

which may be put into the standard form

$$
\tau_{\mathbf{k}, \sigma}^{-1}=\frac{2 \pi}{\hbar} P_{0} \gamma_{\hat{\mathbf{k}}, \sigma}^{2} N^{2} k^{2} n_{\mathbf{k} \sigma} \tanh \left(\frac{\epsilon}{2 k_{\mathrm{B}} T}\right),
$$

where $N=\sqrt{\hbar /(2 V \rho \omega)}$ is the normalization constant of the phonon mode and $\gamma_{\hat{\mathbf{k}}, \sigma} \equiv\left\langle\left|M_{\mathbf{k} \sigma}(\mathfrak{t})\right|^{2}\right\rangle^{1 / 2} /(N k)$ is the (average) phonon-TLS coupling constant. We make the typical assumption that $\widehat{\mathbf{t}}$ is isotropically oriented and the anisotropy is induced only by the properties of $[R]$.

As shown in [19], the structure of $[R]$ should be similar to that of the elastic stiffness constants, $[c]$. The quasicrystal analyzed in [17] has decagonal symmetry and in this case $[R]$ should have the form $[18,26-28]$,

$$
[R]=\left(\begin{array}{cccccc}
r_{11} & r_{12} & r_{13} & 0 & 0 & 0 \\
r_{12} & r_{11} & r_{13} & 0 & 0 & 0 \\
r_{13} & r_{13} & r_{33} & 0 & 0 & 0 \\
0 & 0 & 0 & r_{44} & 0 & 0 \\
0 & 0 & 0 & 0 & r_{44} & 0 \\
0 & 0 & 0 & 0 & 0 & r_{66}
\end{array}\right)
$$

where $r_{66}=\left(r_{11}-r_{12}\right) / 2$ - the axis $z$ is taken along the tenfold axis. The structure of both, $[R]$ and $[c]$, is similar in decagonal quasicrystals and in hexagonal lattices.

Like in hexagonal lattices, in the decagonal quasicrystals, we can have pure longitudinal and transversal waves propagating in all the three directions, $x, y$, and $z$. 
The coupling constants, $\gamma_{\hat{\mathbf{k}}, \sigma}$ are similar to those calculated for hexagonal lattices in [24]:

$$
\begin{gathered}
\gamma_{k \widehat{\mathbf{x}}, l}^{2}=\frac{2\left(r_{11}^{2}+r_{12}^{2}+r_{13}^{2}\right)+\left(r_{11}+r_{12}+r_{13}\right)^{2}}{15}=\gamma_{k \widehat{\mathbf{y}}, l}^{2}, \\
\gamma_{k \widehat{\mathbf{z}}, l}^{2}=\frac{8 r_{13}^{2}+4 r_{13} r_{33}+3 r_{33}^{2}}{15}, \\
\gamma_{k \widehat{\mathbf{y}}, \widehat{\mathbf{x}}, t}^{2}=\gamma_{k \widehat{\mathbf{x}}, \widehat{\mathbf{y}}, t}^{2}=\frac{\left(r_{11}-r_{12}\right)^{2}}{15}=\frac{4 r_{66}^{2}}{15}, \\
\gamma_{k \widehat{\mathbf{x}}, \widehat{\mathbf{z}}, t}^{2}=\gamma_{k \widehat{\mathbf{y}}, \widehat{\mathbf{z}}, t}^{2}=\gamma_{k \widehat{\mathbf{z}}, \widehat{\mathbf{x}}, t}^{2}=\gamma_{k \widehat{\mathbf{z}}, \widehat{\mathbf{y}}, t}^{2}=\frac{4 r_{44}^{2}}{15}
\end{gathered}
$$

where by $l$ and $t$, we refer to longitudinal and transversal polarizations, respectively.

While for the longitudinal waves, the direction of polarization is obvious, for the transversal waves, the direction of polarization is indicated by the second unit vector in the subscript of $\gamma$ in (7c) and (7d).

Due to the isotropy condition in the decagonal plane, the coupling constants of the phonons propagating in this plane are independent of the direction of propagation if they have similar polarization.

Comparing (7c) and (7d) with the results of Bert et al. [18], we obtain

$$
\frac{P \gamma_{\|}^{2}}{P \gamma_{\perp}^{2}} \equiv \frac{\gamma_{k \widehat{\mathbf{x}}, \hat{\mathbf{y}}, t}^{2}}{\gamma_{k \widehat{\mathbf{x}}, \mathbf{\mathrm { z }}, t}^{2}}=\left(\frac{r_{11}-r_{12}}{2 r_{44}}\right)^{2}=\left(\frac{r_{66}}{r_{44}}\right)^{2} \approx 4.2,
$$

where $P \gamma_{\|}^{2}$ and $P \gamma_{\perp}^{2}$ are obvious notations from [18].

To find the ranges of the coupling constants, we rewrite (7a) and (7b) as

$$
\begin{aligned}
\gamma_{k \widehat{\mathbf{x}}, l}^{2}=\gamma_{k \hat{\mathbf{y}}, l}^{2}=\frac{r_{13}^{2}}{80} & {\left[\left(4 \frac{r_{11}}{r_{13}}+1\right)^{2}+\left(4 \frac{r_{12}}{r_{13}}+1\right)^{2}\right.} \\
& \left.+\frac{2}{3}\left(4 \frac{r_{11}}{r_{13}}+1\right)\left(4 \frac{r_{12}}{r_{13}}+1\right)+\frac{40}{3}\right], \\
\gamma_{k \widehat{\mathbf{z}}, l}^{2}= & \frac{r_{13}^{2}}{45}\left[\left(3 \frac{r_{33}}{r_{13}}+2\right)^{2}+20\right] .
\end{aligned}
$$

Since the function $f(a, b)=a^{2}+b^{2}+2 a b / 3$ satisfies $f(a, b) \geq$ $f(0,0)=0$ for any $a$ and $b$, we find from (9a) and (9b) that

$$
\gamma_{k \widehat{\mathbf{x}}, l}^{2}=\gamma_{k \widehat{\mathbf{y}}, l}^{2} \geq \frac{r_{13}^{2}}{6}, \quad \gamma_{k \widehat{\mathbf{z}}, l}^{2} \geq \frac{4 r_{13}^{2}}{9} .
$$

The minimum values of the coupling constants, $\gamma_{k \widehat{\mathbf{x}}, l}^{2}$ and $\gamma_{k \widehat{\mathbf{z}}, l}^{2}$, for given $r_{13}$ (10), are obtained for $r_{11}=r_{12}=-r_{13} / 4$ and $r_{33}=-2 r_{13} / 3$. The ratio between these minimum values is

$$
\frac{\left(\gamma_{k \widehat{\mathbf{x}}, l}^{2}\right)_{\min }}{\left(\gamma_{k \widehat{\mathbf{z}}, l}^{2}\right)_{\min }}=\frac{3}{8}
$$

and is independent of $r_{11}, r_{12}, r_{13}$, and $r_{33}$.
Another inequality exists for the ratio $\gamma_{k \hat{\mathbf{x}}, l}^{2} / \gamma_{k \hat{\mathrm{y}}, \widehat{\mathbf{x}}, t}^{2}$. If we denote $X=r_{12} /\left(r_{11}-r_{12}\right)$ and $Y \equiv r_{13} /\left(r_{11}-r_{12}\right)$, then

$$
\frac{\gamma_{k \widehat{\mathbf{x}}, l}^{2}}{\gamma_{k \widehat{\mathbf{y}}, \widehat{\mathbf{x}}, t}^{2}}=\left(3+8 X^{2}+3 Y^{2}+4 X Y+8 X+2 Y\right) \geq 1
$$

The limit value of the inequality (12), $\gamma_{k \widehat{\mathbf{x}}, l}^{2}=\gamma_{k \widehat{\mathbf{y}}, \widehat{\mathbf{x}}, t}^{2}$, is reached for $X_{\min }=-1 / 2$ and $Y_{\min }=0$.

Relations (11) and (12) are tests for the model, since they should be valid independent of the concrete values of the coupling constants.

\section{The Components of the Tensors}

The anisotropies of the glassy properties of a disordered system are determined by the properties of the tensor $\gamma$. In our model, the "orientations" of the TLSs are defined by the unit vectors $\widehat{\mathbf{t}}$ and the symmetries of the host material are incorporated into the coupling constant tensor, $[R]$. These determine the distributions of the elements of the tensors $\mathrm{T}$ and $\gamma$. This is not possible in the STM. To understand the difficulties to define by general, qualitative arguments the "anisotropy" of $\gamma$ in the STM, we calculate in this section the probability distribution of the elements of our tensor $\mathbf{T}$, under the assumption that the unit vectors $\widehat{\mathbf{t}}$ are isotropically oriented. We will see that neither these elements have all the same distribution of probability, nor the distributions are constant for the ranges of these variables.

We define $\hat{\mathbf{t}}$ by the Euler angles, $\theta$ and $\phi: t_{x}=\sin \theta \cos \phi$, $t_{y}=\sin \theta \sin \phi$, and $t_{z}=\cos \theta$. Keeping $t_{z}$ and $\phi$ as variables, we write $\mathbf{T}^{t}=\left[\left(1-t_{z}^{2}\right) \cos ^{2} \phi,\left(1-t_{z}^{2}\right) \sin ^{2} \phi, t_{z}^{2}, 2 t_{z} \sqrt{1-t_{z}^{2}} \sin \phi\right.$, $\left.2 t_{z} \sqrt{1-t_{z}^{2}} \cos \phi,\left(1-t_{z}^{2}\right) \sin (2 \phi)\right]$.

The variables $t_{z}$ and $\phi$ are uniformly distributed in the intervals $[0,1]$ and $[0,2 \pi]$, respectively. We denote their probability distribution by $P_{\widehat{\mathbf{t}}}\left(t_{z}, \phi\right)=1 /(4 \pi)$. Then the probability distribution in the variables $T_{I}(I=1, \ldots, 6)$ and $\phi$ is

$$
P_{I, \phi}\left(T_{I}, \phi\right)=\frac{1}{4 \pi}\left|\frac{\partial\left(t_{z}, \phi\right)}{\partial\left(T_{I}, \phi\right)}\right|=\frac{1}{4 \pi}\left|\frac{\partial\left(T_{I}, \phi\right)}{\partial\left(t_{z}, \phi\right)}\right|^{-1}
$$

The simplest of all is eventually

$$
P_{3, \phi}\left(T_{3}, \phi\right)=\frac{1}{4 \pi \sqrt{T_{3}}}
$$

which, if integrated over $\phi$, gives

$$
P_{3}\left(T_{3}\right)=\frac{1}{2 \sqrt{T_{3}}}
$$




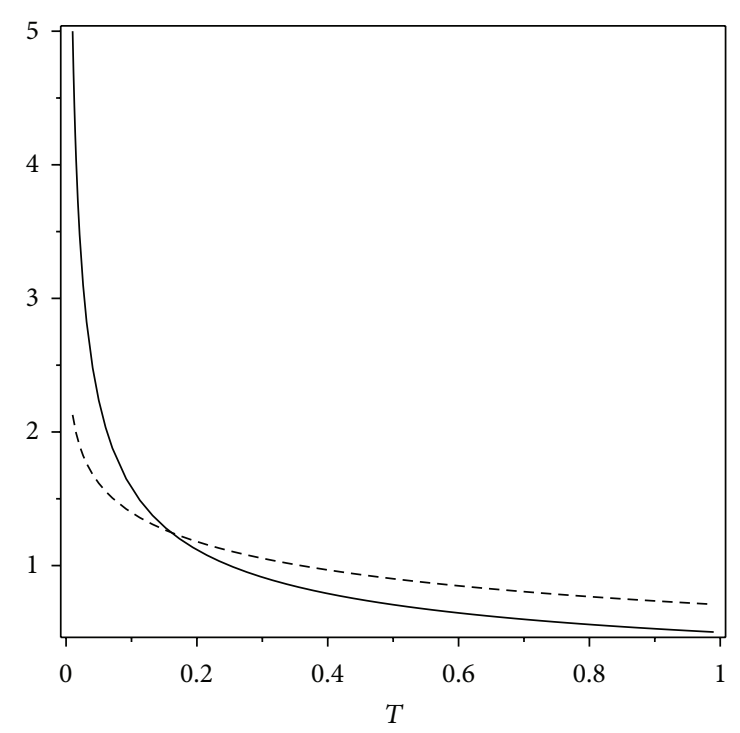

FIgURE 1: The probability distributions of the components of the tensor T: $P_{1}(T)=P_{2}(T)=P_{3}(T)=1 /(2 \sqrt{T})$, for $T \in(0,1]$ (solid line), and $2 P_{4}(|T|)=2 P_{5}(|T|)=2 P_{6}(|T|)$, for $T \in[-1,0) \cup(0,1]$ (dashed line).

For the components $T_{1}$ and $T_{2}$, we obtain the probability distributions

$$
\begin{aligned}
& P_{1, \phi}\left(T_{1}, \phi\right)=\frac{1}{4 \pi} \frac{\theta\left(\cos ^{2} \phi-T_{1}\right)}{\sqrt{1-\left(T_{1} / \cos ^{2} \phi\right)} \cos ^{2} \phi} \\
& P_{2, \phi}\left(T_{2}, \phi\right)=\frac{1}{4 \pi} \frac{\theta\left(\sin ^{2} \phi-T_{2}\right)}{\sqrt{1-\left(T_{2} / \sin ^{2} \phi\right)} \sin ^{2} \phi}
\end{aligned}
$$

where $\theta(x)$ is the Heaviside step function. An extra factor of 2 appears in (16a) and (16b) because $T_{1}$ and $T_{2}$ are even functions of $t_{z}$.

For an isotropic distribution of $\widehat{\mathfrak{t}}$, the components $T_{1}, T_{2}$, and $T_{3}$ are equivalent, and using (15) and (16a) and (16b), we obtain the identity (Figure 1, solid line)

$$
P_{1}(T)=P_{2}(T)=\frac{1}{\pi} \int_{\sqrt{T}}^{1} \frac{d x}{x \sqrt{\left(x^{2}-T_{1}\right)\left(1-x^{2}\right)}} \equiv \frac{1}{2 \sqrt{T}} .
$$

The components $T_{4}, T_{5}$, and $T_{6}$ are also equivalent to isotropic $\widehat{\mathbf{t}}$. For example,

$$
P_{6, \phi}\left(T_{6}, \phi\right)=\frac{1}{4 \pi} \frac{\theta\left(|\sin (2 \phi)|-\left|T_{6}\right|\right)}{\sqrt{1-T_{6} / \sin (2 \phi)}|\sin (2 \phi)|},
$$

where $T_{6} / \sin (2 \phi)=1-t_{z}^{2} \geq 0$. From (18a) we get

$$
\begin{aligned}
P_{6}(T)= & \frac{1}{2 \pi} \int_{|T|}^{1} \frac{d x}{\sqrt{x(x-T)\left(1-x^{2}\right)}} \\
& =P_{4}(T)=P_{5}(T),
\end{aligned}
$$

where $T \in[-1,1]$ and $P_{4}(|T|)=P_{5}(|T|)=P_{6}(|T|)$ (Figure 1, dashed line).

We notice that $\lim _{T \rightarrow 1} P_{T_{6}}(T)=1 /(2 \sqrt{2})$, whereas in 0 , $P_{T_{6}}(T)$ has a logarithmic divergence.

The equalities (18c) are obtained using the equivalence between the components $T_{4}, T_{5}$, and $T_{6}$.

We observe that the isotropic distribution of the TLS orientations does not correspond to a constant distribution of the values of the components of $\mathbf{T}$, nor to equal distributions of these values. Therefore it is not straightforward to draw conclusions regarding the relations between the distributions of the values of $\gamma_{I}$, based only on general arguments.

\section{Conclusions}

In this paper we describe the anisotropy of the glassy properties of the decagonal quasicrystals in the model of [19]. We show that the glassy properties of these quasicrystals are similar to those of hexagonal disordered lattices [24] and we obtained the TLS-phonon average coupling constants, $\gamma_{\mathbf{k}, \sigma}^{2}$, which are dependent on the phonon's propagation direction, $\mathbf{k}$, and polarization, $\sigma$. We apply the results to the experimental data of [18] and we obtain the ratio $\left(r_{66} / r_{44}\right)^{2} \approx$ 4.2, where $r_{44}$ and $r_{66}$ are components of the tensor of coupling constants.

In order to better understand the characteristics of "isotropic" and "anisotropic" distributions of TLSs, we calculated the probability distributions of the elements of the tensor $\mathbf{T}$ - which describes the TLS - under the assumption that the directions of the ensemble of TLSs, defined by the unit vectors $\widehat{\mathbf{t}}$, are isotropically oriented. We observe that the distributions of the elements of $\mathbf{T}$ are rather complicated and therefore they cannot be found in the STM, simply based on qualitative, general arguments.

\section{Acknowledgments}

The work was supported by the Romanian National Authority for Scientific Research projects PN-II-ID-PCE-2011-3-0960 and PN09370102/2009. The travel support from the RomaniaJINR Dubna collaboration project Titeica-Markov and project N4063 is gratefully acknowledged.

\section{References}

[1] R. C. Zeller and R. O. Pohl, "Thermal conductivity and specific heat of noncrystalline solids," Physical Review B, vol. 4, no. 6, pp. 2029-2041, 1971.

[2] P. Esquinazi, Tunneling Systems in Amorphous and Crystalline Solids, Springer, Berlin, Germany, 1998. 
[3] R. O. Pohl, X. Liu, and E. Thompson, "Low-temperature thermal conductivity and acoustic attenuation in amorphous solids," Reviews of Modern Physics, vol. 74, no. 4, pp. 991-1013, 2002.

[4] M. I. Klinger, "Soft atomic motion modes in glasses: their role in anomalous properties," Physics Reports, vol. 492, no. 4-5, pp. $111-180,2010$.

[5] L. F. Lou, “Glassy' low-temperature thermal properties in crystalline superconducting $\mathrm{Zr}-\mathrm{Nb}$ alloys," Solid State Communications, vol. 19, pp. 335-338, 1976.

[6] P. Doussineau, C. Frenois, R. G. Leisure, A. Levelut, and J. Y. Prieur, "Amorphous-like acoustical properties of na doped beta $-\mathrm{Al}_{2} \mathrm{O}_{3}$," Journal de Physique, vol. 41, no. 10, pp. 1193-1211, 1980.

[7] A. Vanelstraete and C. Laermans, "Tunneling states in neutronirradiated quartz: measurements of the ultrasonic attenuation and velocity change," Physical Review B, vol. 42, no. 9, pp. 5842$5854,1990$.

[8] C. Laermans and V. Keppens, "Origin of the tunneling states in $\mathrm{SiO}_{2}$," Physical Review B, vol. 51, no. 13, pp. 8158-8163, 1995.

[9] X. Liu, P. D. Vu, R. O. Pohl, F. Schiettekatte, and S. Roorda, "Generation of low-energy excitations in silicon," Physical Review Letters, vol. 81, no. 15, pp. 3171-3174, 1998.

[10] K. A. Topp, Effects of random strains on tunneling states in crystals [Ph.D. thesis], 1997.

[11] S. K. Watson, "Tunneling states in crystals with large random strains," Physical Review Letters, vol. 75, no. 10, pp. 1965-11968, 1995.

[12] K. A. Topp and D. G. Cahill, "Elastic properties of several amorphous solids and disordered crystals below 100K," Zeitschrift für Physik B, vol. 101, pp. 235-245, 1996.

[13] K. A. Topp, E. Thompson, and R. O. Pohl, "Glasslike excitations in chemically disordered crystals: alkali-earth lanthanum fluoride mixed crystals," Physical Review B, vol. 60, no. 2, pp. 898908, 1999.

[14] E. J. Thompson, P. D. Vu, and R. O. Pohl, "Glasslike lattice vibrations in the quasicrystal $\mathrm{Al}_{72.1} \mathrm{Pd}_{20.7} \mathrm{Mn}_{7.2}$," Physical Review $B$, vol. 62, pp. 11437-11443, 2000.

[15] K. Giannò, A. V. Sologubenko, M. A. Chernikov, H. R. Ott, I. R. Fisher, and P. C. Canfield, "Low-temperature thermal conductivity of a single-grain Y-Mg-Zn icosahedral quasicrystal," Physical Review B, vol. 62, pp. 292-300, 2000.

[16] F. Bert and G. Bellessa, "Tunneling states in Al-Li-Cu quasicrystals," Physical Review B, vol. 65, no. 1, Article ID 014202, 5 pages, 2001.

[17] F. Bert, G. Bellessa, A. Quivy, and Y. Calvayrac, "Tunneling states in a single-grain Al-Cu-Fe quasicrystal," Physical Review $B$, vol. 61, no. 1, pp. 32-35, 2000.

[18] F. Bert, G. Bellessa, and B. Grushko, "Tunneling state anisotropy in a single grain decagonal quasicrystal," Physical Review Letters, vol. 88, Article ID 255901, 4 pages, 2002.

[19] D. V. Anghel, T. Kühn, Y. M. Galperin, and M. Manninen, "Interaction of two-level systems in amorphous materials with arbitrary phonon fields," Physical Review B, vol. 75, Article ID 064202, 6 pages, 2007.

[20] T. Küuhn, D. V. Anghel, Y. M. Galperin, and M. Manninen, "Interaction of Lamb modes with two-level systems in amorphous nanoscopic membranes," Physical Review B, vol. 76, Article ID 165425, 9 pages, 2007.

[21] D. V. Anghel, T. Kühn, and M. Manninen, "The tensor of interaction of a two-level system with an arbitrary strain field," Journal of Physics Conference Series, vol. 92, Article ID 012133, 2007.
[22] D. V. Anghel and D. V. Churochkin, "Anisotropic interaction of two-level systems with acoustic waves in disordered cubic crystals," Physical Review B, vol. 78, Article ID 94202, 5 pages, 2008.

[23] D. V. Anghel and D. V. Churochkin, "The anisotropic glass-like properties of disordered crystals," Journal of Physics Conference Series, vol. 150, part 1, Article ID 012002, 2008.

[24] D. V. Anghel and D. Churochkin, "Scattering of phonons on two-level systems in disordered crystals," Europhysics Letters, vol. 83, no. 5, Article ID 56004, 2008.

[25] D. V. Anghel, "Properties of two-level systems in disordered materials," Romanian Journal of Physics, vol. 55, pp. 903-912, 2010.

[26] M. A. Chernikov, "Elastic properties of icosahedral and decagonal quasicrystals," Physics-Uspekhi, vol. 48, no. 4, p. 411, 2005.

[27] C. L. Henley, M. Mihalkovič, and M. Widom, "Total-energybased structure prediction for d(AlNiCo)," Journal of Alloys and Compounds, vol. 342, no. 1-2, pp. 221-227, 2002.

[28] M. Mihalkovič and M. Widom, "Tile decoration model of the W-(Al-Co-Ni) approximant," Philosophical Magazine, vol. 86, pp. $557-565,2006$. 

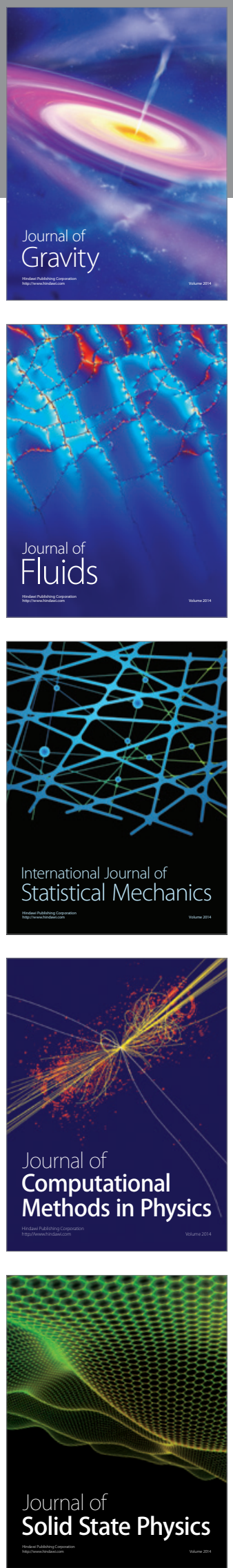

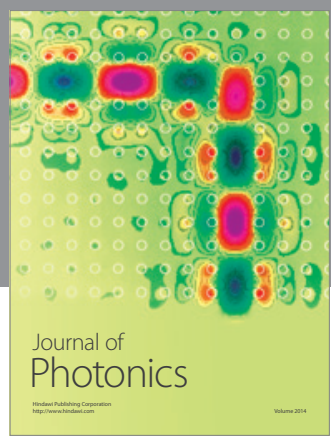

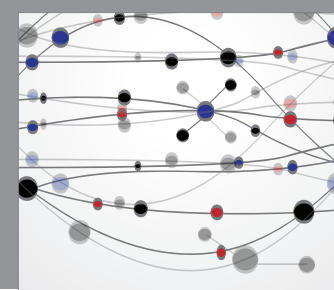

The Scientific World Journal

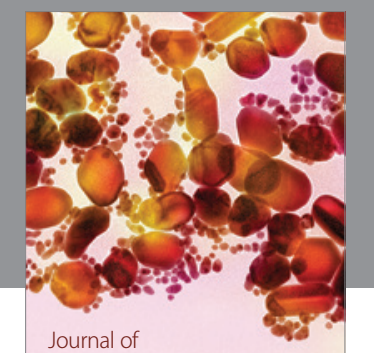

Soft Matter
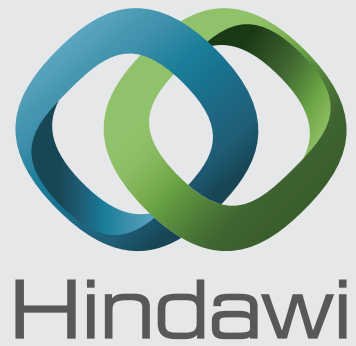

Submit your manuscripts at

http://www.hindawi.com
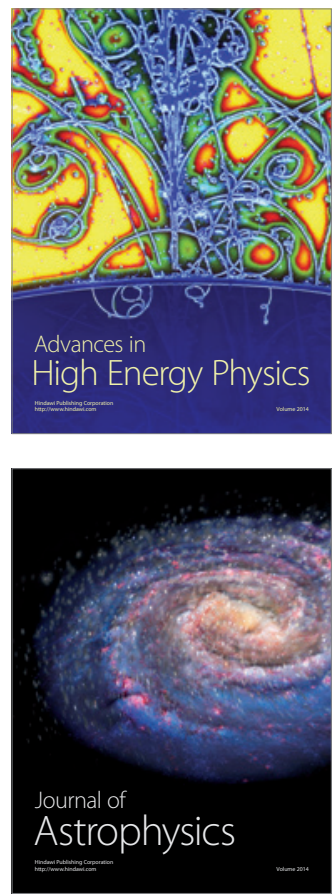
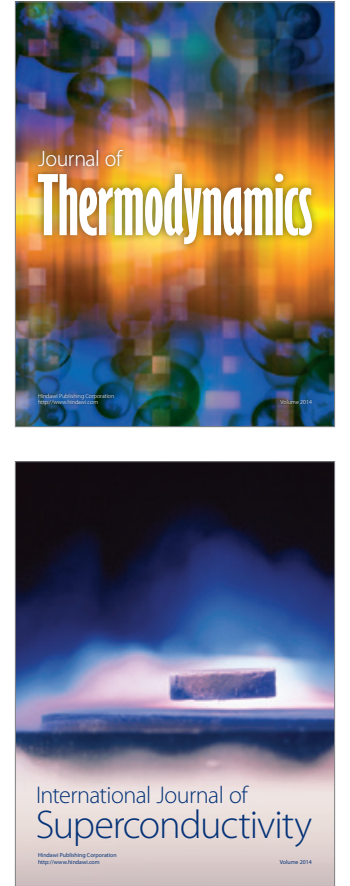
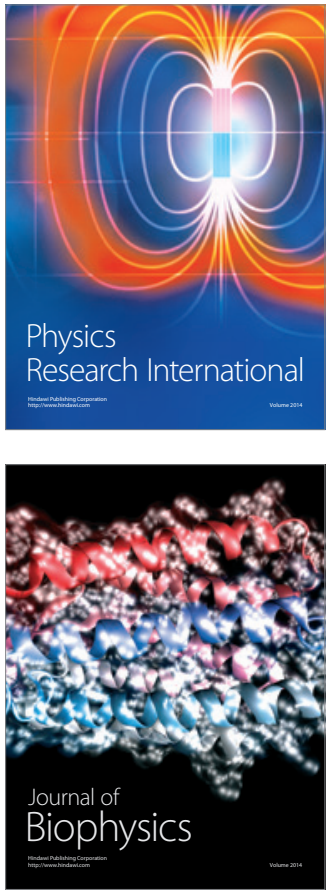
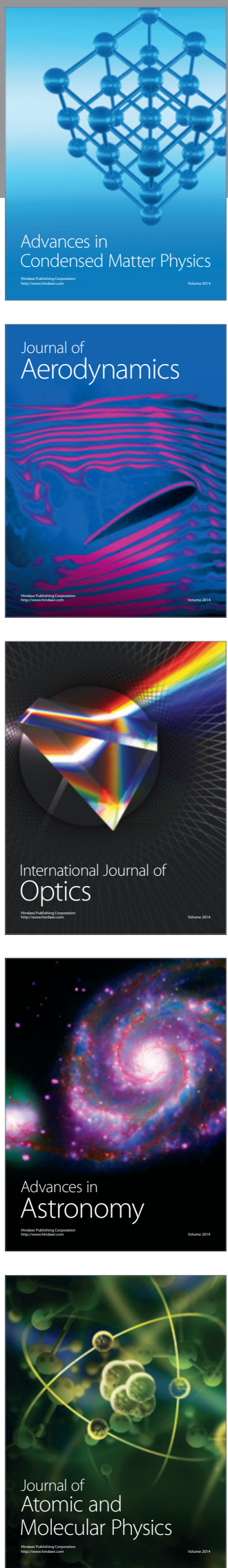